\title{
Fall detection analysis using a real fall dataset*
}

\author{
Samad Barri Khojasteh ${ }^{1,2}$, José R. Villar², Enrique de la Cal², Víctor M. \\ González $^{3}$, and Javier Sedano ${ }^{4}$ \\ 1 Sakarya University, Department of Industrial Engineering, \\ Sakarya, Turkey \\ samad.khojasteh@ogr.sakarya.edu.tr, samad.khojasteh@gmail.com \\ 2 University of Oviedo, Computer Science Department, \\ EIMEM, Oviedo, Spain \\ $\{$ villarjose, delacal\}@uniovi.es \\ 3 University of Oviedo, Control and Automatica Department, \\ EPI, Gijón, Spain \\ vmsuarez@uniovi.es \\ 4 Instituto Tecnológico de Castilla y León, Pol. Ind. Villalonquejar, \\ 09001, Burgos, Spain \\ javier.sedano@itcl.es
}

\begin{abstract}
This study focuses on the performance of a fall detection method using data coming from real falls performed by relatively young people and the application of this technique in the case of an elder person. Although the vast majority of studies concerning fall detection place the sensory on the waist, in this research the wearable device must be placed on the wrist because it's usability. A first pre-processing stage is carried out as stated in 1117; this stage detects the most relevant points to label. This study analyzes the suitability of different models in solving this classification problem: a feed-forward Neural Network and a rule based system generated with the C5.0 algorithm. A discussion about the results and the deployment issues is included
\end{abstract}

\section{INTRODUCTION}

Fall Detection (FD) is a very active research area, with many applications to healthcare, work safety, etc. Even though there are plenty of commercial products, the best rated products only reach a $80 \%$ of success 20 . There are basically two types of FD systems: contex-aware systems and wearable devices 14. FD has been widely studied using context-aware systems, i.e. video systems [28; nevertheless, the use of wearable devices is crucial because the high percentage of eldel people and their desire to live autonomously in their own house [19].

Wearables-based solutions may combine different sensors, such as a barometer and inertial sensors 22, 3DACC and gyroscope [23, 3DACC and intelligent tiles [7] or a 3DACC and a barometer in a necklace was also reported in [3]. However, 3DACC is by far the most chosen option 29]12 27/4]13, with a variable number of sensors and locations, even some of them proposed the use of the smartphone sensory system. Different solutions have been proposed to perform 
the fall event detection, for instance, a feature extraction stage and Support Vector Machines have been applied directly in 2927, using some transformations and thresholds with very simple rules for classifying an event as a fall [4]13 16]. A comparison of classifiers has been presented in 12, comparing Decision tree, SVM, Nearest neghborn and Discrimenent analysis. Several threshold-based fall detection algorithms were presented in 4|910. The two latter employed three threshold algorithms based to compare with the acceleration magnitude. Igual et al. compared several public datasets for fall detection via support machine vector (SVM) and nearest neighbor (NN) and analyzed results them [15. The common characteristic in all these solutions is that the wearable devices are placed on the waist or in the chest. This research limits itself to use a single sensor -a marketed smartwatch- placed on the wrist in order to promote its usability.

Interestingly, the previous studies do not focus on the specific dynamics of a falling event: although some of the proposals report good performances, they are just machine learning applied to the focused problem. There are studies concerned with the dynamics in a fall event [28, establishing the taxonomy and the time periods for each sequence. Additionally, Abbate et al proposed the use of these dynamics as the basis of the FD algorithm [1. A very interesting point of this approach is that the computational constraints are kept moderate, although this solution includes a high number of thresholds to tune. In [17, this solution was analyzed with data gathered from sensors placed on the wrist, using the Abate solution plus a SMOTE balancing stage and a feed-forward Neural Network. In this research, an alternative based on C5.0 rule based systems is proposed.

\section{Adapting Fall detection to a wrist-based solution}

Abate et al 1 proposed the following scheme to detect a candidate event as a fall event (refer to Fig. 1). A time $t$ corresponds to a peak time (point 1 ) if the magnitude of the acceleration $a$ is higher than $t h_{1}=3 \times g, g=9.8 \mathrm{~m} / \mathrm{s}$. After a peak time there must be a period of $2500 \mathrm{~ms}$ with relatively calm (no other $a$ value higher than $t h_{1}$ ). The impact end (point 2) denotes the end of the fall event; it is the last time for which the $a$ value is higher than $t h_{2}=1.5 \times g$. Finally, the impact start (point 3) denotes the starting time of the fall event, computed as the time of the first sequence of an $a<=t h_{3}\left(t h_{3}=0.8 \times g\right)$ followed by a value of $a>=t h_{2}$. The impact start must belong to the interval [impact end-1200 ms, peak time]. If no impact end is found, then it is fixed to peak time plus $1000 \mathrm{~ms}$. If no impact start is found, it is fixed to peak time.

Whenever a peak time is found, the following transformations should be computed:

- Average Absolute Acceleration Magnitude Variation, $A A M V=\sum_{t=i s}^{i e} \frac{\left|a_{t+1}-a_{t}\right|}{N}$, with $i s$ being the impact start, ie the impact end, and $N$ the number of samples in the interval.

- Impact Duration Index, IDI = impact end - impact start. Alternatively, it could be computed as the number of samples. 
- Maximum Peak Index, $M P I=\max _{t \in[i s, i e]}\left(a_{t}\right)$.

- Minimum Valley Index, $M V I=\min _{t \in[i s-500, i e]}\left(a_{t}\right)$.

- Peak Duration Index, PDI = peak end-peak start, with peak start defined as the time of the last magnitude sample below $t h_{P D I}=1.8 \times g$ occurred before peak time, and peak end is defined as the time of the first magnitude sample below $t h_{P D I}=1.8 \times g$ occurred after peak time.

- Activity Ratio Index, ARI, measuring the activity level in an interval of 700 ms centered at the middle time between impact start and impact end. The activity level is calculated as the ratio between the number of samples not in $\left[h_{A R I l o w} 0.85 \times g, t h_{A R I I h i g h}=1.3 \times g\right]$ and the total number of samples in the $700 \mathrm{~ms}$ interval.

- Free Fall Index, FFI, computed as follows. Firstly, search for an acceleration sample below $t h_{F F I}=0.8 \times g$ occurring up to $200 \mathrm{~ms}$ before peak time; if found, the sample time represents the end of the interval, otherwise the end of the interval is set $200 \mathrm{~ms}$ before peak time. Secondly, the start of the interval is simply set to $200 \mathrm{~ms}$ before its end. FFI is defined as the average acceleration magnitude evaluated within the interval.

- Step Count Index, $S C I$, measured as the number of peaks in the interval [peak time-2200, peak time]. SCI is the step count evaluated $2200 \mathrm{~ms}$ before peak time. The number of valleys are counted, defining a valley as a region with acceleration magnitude below $t h_{S C I l o w}=1 \times g$ for at least $80 \mathrm{~ms}$, followed by a magnitude higher than $t h_{S C I h i g h} 1.6 \times g$ during the next 200 ms. Some ideas on computing the time between peaks [26] were used when implementing this feature.

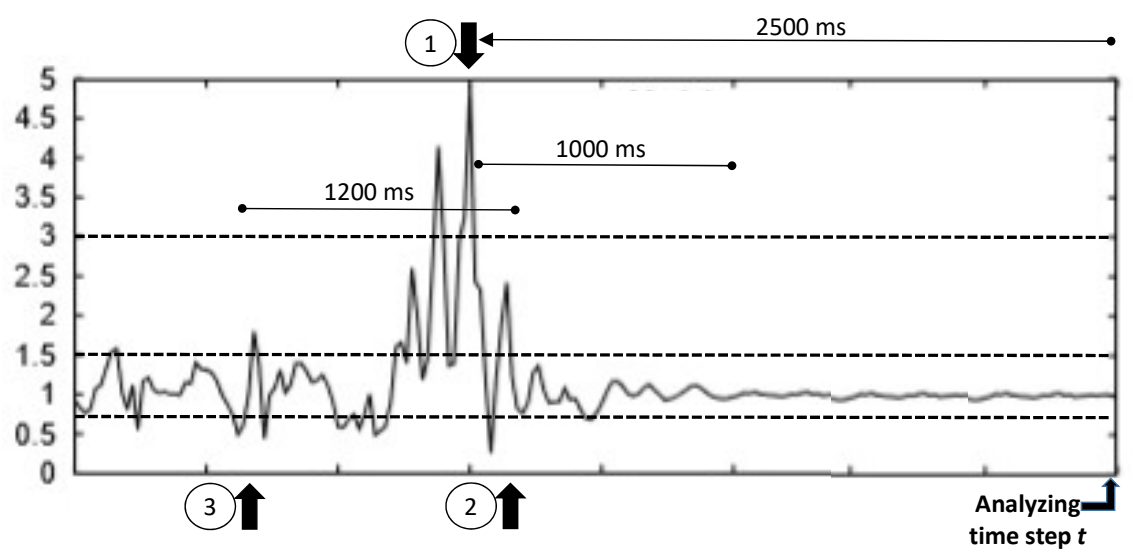

Fig. 1. Evolution of the magnitude of the acceleration -y-axis, extracted from [1].

Evaluating this approach was proposed as follows. The time series of acceleration magnitude values are analyzed searching for peaks that marks where a fall event candidate appears. When it happens to occur, the impact end and the 
impact start are determined, and thus the remaining features. As long as this fall events are detected when walking or running, for instance, a Neural Network $(\mathrm{NN})$ model is obtained to classify the set of features extracted.

In order to train the $\mathrm{NN}$, the authors made use of an Activities of Daily Living (ADL) and FD dataset, where each file contains a Time Series of 3DACC values corresponding to an activity or to a fall event. Therefore, each dataset including a fall event or a similar activity -for instance, running can perform similarly to falling- will generate a set of transformation values. Thus, for a dataset file we will detect something similar to a falling, producing a row of the transformations computed for each of the detected events within the file. If nothing is detected withing the file, no row is produced. With this strategy, the Abbate et al obtained the training and testing dataset to learn the NN.

\subsection{The modifications on the algorithm}

As stated in 11|25, the solutions to this type of problems must be ergonomic: the users must feel comfortable using them. We considered that placing a device on the waist is not comfortable, for instance, it is not valid for women using dresses. When working with elder people, this issue is of main relevance. Therefore, in this study, we placed the wearable device on the wrist. This is not a simple change: the vast majority of the literature reports solutions for FD using waist based solutions. Moreover, according to 24 the calculations should be performed on the smartwatches to extend the battery life by reducing the communications. Therefore, these calculations should be kept as simple as possible.

A second modification is focused on the training of the NN. The original strategy for the generation of the training and testing dataset produced a highly imbalanced dataset: up to $81 \%$ of the obtained samples belong to the class FD, while the remaining belong to the different ADL similar to a fall event.

To solve this problem a normalization stage is applied to the generated imbalanced dataset, followed by a SMOTE balancing stage [6. This balancing stage will produce a $60 \%$ (FALL)- $40 \%$ (no FALL) dataset, which would allow to avoid the over-fitting of the NN models. As usual, there is a compromise between the balancing of the dataset and the synthetic data samples introduced in the dataset.

These above mentioned changes have already been studied in [17. In this research we proposed to analyze the performance of rule based systems in this context, which represents more simpler models that can be easily deployed in wearable devices and with a very reduced computational complexity. Therefore, they could represent a very interesting improvement, either if they work similarly to the NN or just similarly to them.

\section{$3 \quad$ Experiments and results}

A ADL and FD dataset is needed to evaluate the adaptation, so it contains time series sample from ADL and for falls. This research made use of the UMA-FALL 
dataset [5] among the publicly available datasets. This dataset includes data for several participants carrying on with different activities and performing forward, backward and lateral falls. Actually, this falls are not real falls -demonstrative videos have been also published-, but they can represent the initial step for evaluating the adapted solution problem. Interestingly, this dataset includes multiple sensors; therefore, the researcher can evaluate the approach using sensors placed on different parts of the body.

The thresholds used in this study are exactly the same as those mentioned in the original paper. All the code was implemented in R21 and caret 18. The parameters for SMOTE were perc.over set to 300 and perc.under set to 200 -that is, 3 minority class samples are generated per original sample while keeping 2 samples from the majority class-. These parameters produces a balanced dataset that moves from a distribution of 47 samples from the minority class and 200 from the majority class to a 188 minority class versus 282 majority class (40\%/60\% of balance).

To obtain the parameters for the NN a grid search was performed; the final values were size set to 20 , decay set to $10^{-3}$ and maximum number of iterations 500 , the absolute and relative tolerances set to $4 \times 10^{-6}$ and $10^{-10}$, respectively. In this research, we use the C5.0 implementation of the $\mathrm{C} 4.5$ that is included in the $\mathrm{R}$ package to obtain the rule based systems. The parameters found optimum for this classification problem are cf set to 0.25 , bands set to 2 , the fuzzy Threshold parameter set to TRUE, the number of trials set to 15 , and winnow set to FALSE.

Both $5 \times 2$ cross validation (cv) and 10-fold cv were performed to analyzed the robustness of the solution. The latter cv would allow us to compare with existing solutions, while the former shows the performance of the system with an increase in the number of unseen samples. The results are shown in Table 1 and Table 2 for 10 -fold cv and $5 \times 2 \mathrm{cv}$, respectively.

\subsection{Discussion on the results}

From the tables it can be seen that both modelling techniques perform exceptionally well once the SMOTE is performed and using test folds from 10-fold cv: the models even perform ideally for several folds. And more importantly, the two models are interchangeable with no apparent loss in the performance. Actually, these results are rather similar to those published in the orignal work [1. However, when using $5 \times 2 \mathrm{cv}$ the results diverts from those previously mentioned.

With $5 \times 2 \mathrm{cv}$, the size of the train and test datasets are of similar number of samples, producing a worse training and, what is more interesting, introduces more variability in the test dataset. Therefore, the results are worse. The point is that these results suggest the task is not solved yet as the number of false alarms increased unexpectedly.

This problem is important because in this experimentation we used the UMAFall dataset [5]. This dataset used was generated with young participants using a very deterministic protocol of activities. The falls were performed with the participants standing still and letting them fall in the forward/backward/lateral 


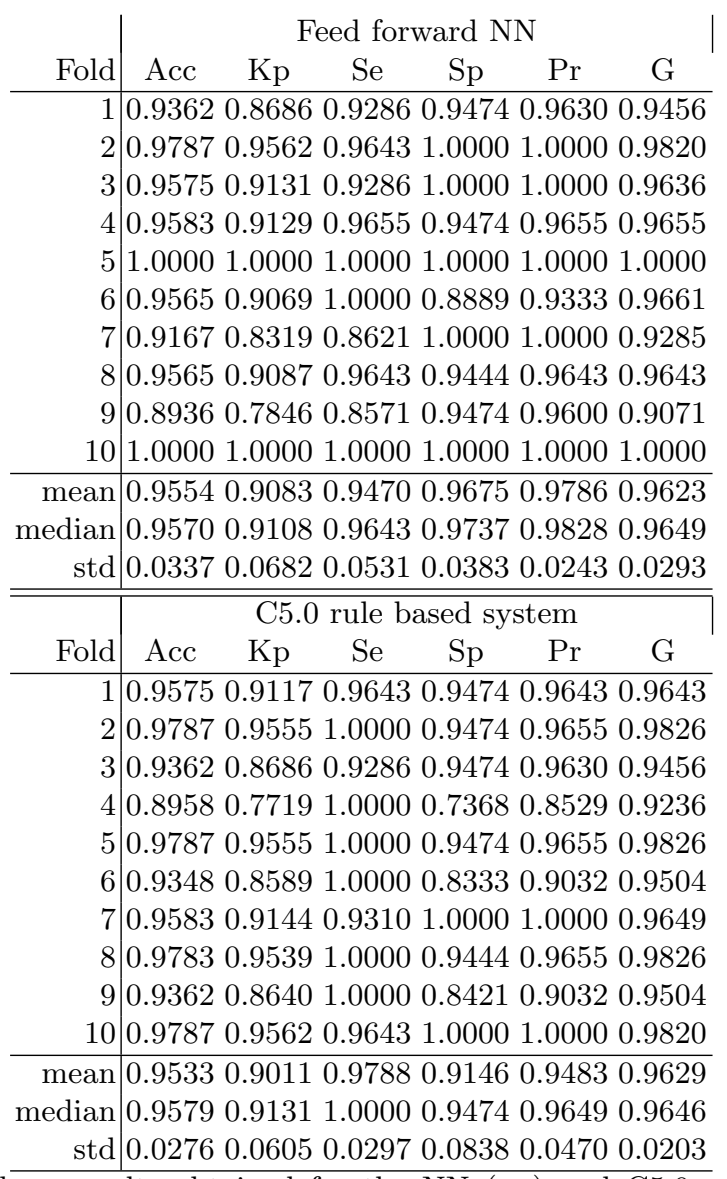

Table 1. 10 fold $\mathrm{cv}$ results obtained for the NN (up) and C5.0 rule based system (bottom). From left to right, the main statistical measurements are shown: accuracy (Acc), Kappa factor (Kp, sensitivity (Se), the specificity (Sp), the precision $(\mathrm{Pr})$ and the geometric mean of the Acc and $\operatorname{Pr}, G=\sqrt[2]{\operatorname{Pr} \times A c c}$. 


\begin{tabular}{r||lccccc} 
& \multicolumn{5}{|c}{ Feed forward NN } \\
Fold & Acc & Kp & Se & Sp & Pr & G \\
\hline 1 & 0.8936 & 0.7772 & 0.9220 & 0.8511 & 0.9028 & 0.9123 \\
2 & 0.9319 & 0.8571 & 0.9575 & 0.8936 & 0.9310 & 0.9442 \\
3 & 0.9192 & 0.8359 & 0.8794 & 0.9787 & 0.9841 & 0.9303 \\
4 & 0.9532 & 0.9027 & 0.9575 & 0.9468 & 0.9643 & 0.9609 \\
5 & 0.9362 & 0.8682 & 0.9291 & 0.9468 & 0.9632 & 0.9460 \\
6 & 0.9106 & 0.8148 & 0.9149 & 0.9043 & 0.9348 & 0.9248 \\
7 & 0.9362 & 0.8682 & 0.9291 & 0.9468 & 0.9632 & 0.9460 \\
8 & 0.9404 & 0.8750 & 0.9645 & 0.9043 & 0.9379 & 0.9511 \\
9 & 0.9149 & 0.8252 & 0.9007 & 0.9362 & 0.9549 & 0.9274 \\
10 & 0.9021 & 0.7935 & 0.9433 & 0.8404 & 0.8987 & 0.9207 \\
\hline mean & 0.9238 & 0.8418 & 0.9298 & 0.9149 & 0.9435 & 0.9364 \\
median & 0.9255 & 0.8465 & 0.9291 & 0.9202 & 0.9464 & 0.9372 \\
std & 0.0188 & 0.0394 & 0.0271 & 0.0446 & 0.0277 & 0.0154 \\
\hline \hline Fold & \multicolumn{7}{|c|}{ Acc } & Kp & Se & Sp & Pr & rule based & system & \\
\hline 1 & 0.9234 & 0.8387 & 0.9575 & 0.8723 & 0.9184 & 0.9377 \\
2 & 0.9362 & 0.8654 & 0.9716 & 0.8830 & 0.9257 & 0.9484 \\
3 & 0.9149 & 0.8214 & 0.9433 & 0.8723 & 0.9172 & 0.9302 \\
4 & 0.9404 & 0.8746 & 0.9716 & 0.8936 & 0.9320 & 0.9516 \\
5 & 0.9319 & 0.8556 & 0.9787 & 0.8617 & 0.9139 & 0.9458 \\
6 & 0.9106 & 0.8087 & 0.9787 & 0.8085 & 0.8846 & 0.9305 \\
7 & 0.8894 & 0.7679 & 0.9220 & 0.8404 & 0.8966 & 0.9092 \\
8 & 0.9362 & 0.8649 & 0.9787 & 0.8723 & 0.9200 & 0.9489 \\
9 & 0.9149 & 0.8162 & 1.0000 & 0.7872 & 0.8758 & 0.9358 \\
10 & 0.9021 & 0.7898 & 0.9787 & 0.7872 & 0.8734 & 0.9246 \\
\hline mean & 0.9200 & 0.8303 & 0.9681 & 0.8479 & 0.9058 & 0.9363 \\
median & 0.9192 & 0.8301 & 0.9752 & 0.8670 & 0.9156 & 0.9368 \\
std & 0.0166 & 0.0356 & 0.0220 & 0.0398 & 0.0214 & 0.0132 \\
\hline
\end{tabular}

Table 2. $5 \times 2 \mathrm{cv}$ results obtained for the NN (up) and C5.0 rule based system (bottom). From left to right, the main statistical measurements are shown: accuracy (Acc), Kappa factor (Kp, sensitivity (Se), the specificity $(\mathrm{Sp})$, the precision $(\mathrm{Pr})$ and the geometric mean of the Acc and $\operatorname{Pr}, G=\sqrt[2]{\operatorname{Pr} \times A c c}$. 


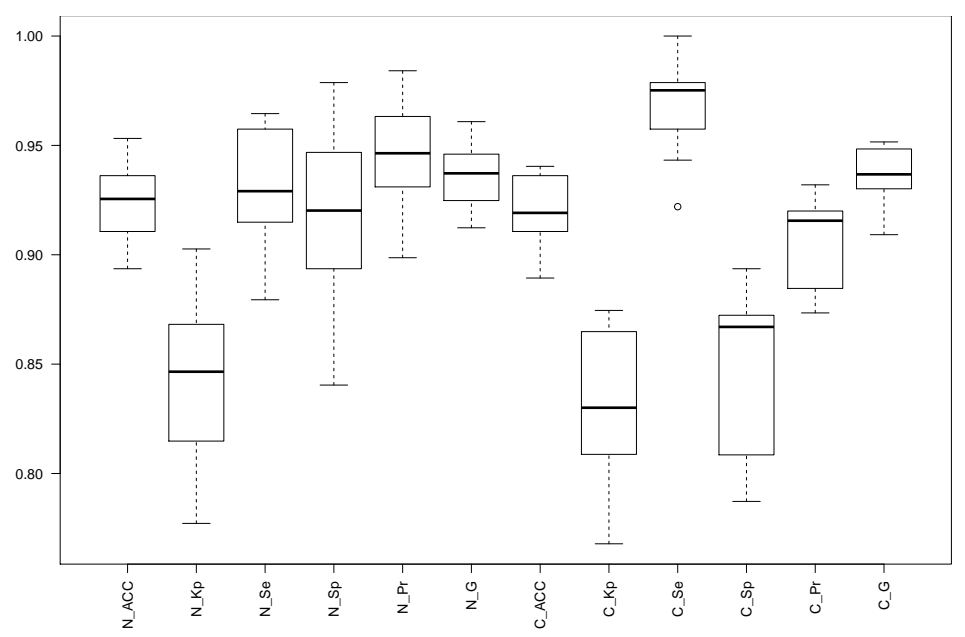

Fig. 2. $5 \times 2 \mathrm{cv}$ Boxplot for the different measurements -Accuracy (Acc), Kappa (Kp), Sensitivity (Se) and Specificity (Sp), Precision (Pr) and the geometric mean of the Acc and $\operatorname{Pr}, G=\sqrt[2]{\operatorname{Pr} \times A c c}$-, both for the feed-forward NN (six boxplots to the left, with the $\mathrm{N}_{-}$prefix) and C5.0 (six boxplots to the right, with the $\mathrm{C}_{-}$prefix).

direction. Therefore, the differences with real falls might be relevant; even if they are not so different, the variability that might be introduced will severely punish the performance of the obtained models.

\section{Conclusions}

This study compares the performances of two classification techniques when tackling the problem fall detection with data gathered from accelerometers located on one wrist. The original proposal detected fall events and performed a feature extraction which was classified with a feed-forward NN. A SMOTE stage is included to balance the transformed dataset previous modelling. Two different techniques are compared: the feed-forward $\mathrm{NN}$ and $\mathrm{C} 5.0$ rule based systems. A publicly available dataset with falls has been used in evaluating the proposal. Interestingly, the two modelling techniques performed similarly, which suggest that in real world applications with the solution embedded in smartwatches perhaps the rule based systems is more likely to be used.

Although exceptional results have been found using 10 fold cv, the $5 \times 2 \mathrm{cv}$ results suggest that still a high number of false alarms is obtained. Although the percentages are better that those reported for commercial devices, some design aspects must be analyzed in depth: the robustness to the variability in the behaviour of the user, or the tuning of the threshold to fit specific populations like the elderly. 


\section{ACKNOWLEDGMENT}

This research has been funded by the Spanish Ministry of Science and Innovation, under project MINECO-TIN2014-56967-R and MINECO-TIN2017-84804R, and FC-15-GRUPIN14-073 (Regional Ministry of Principality of Asturias).

\section{References}

1. Abbate, S., Avvenuti, M., Bonatesta, F., Cola, G., Corsini, P., AlessioVecchio: A smartphone-based fall detection system. Pervasive and Mobile Computing 8(6), 883-899 (Dec 2012)

2. Abbate, S., Avvenuti, M., Corsini, P., Light, J., Vecchio, A.: Wireless Sensor Networks: Application - Centric Design, chap. Monitoring of human movements for fall detection and activities recognition in elderly care using wireless sensor network: a survey, p. 22. Intech (2010)

3. Bianchi, F., Redmond, S.J., Narayanan, M.R., Cerutti, S., Lovell, N.H.: Barometric pressure and triaxial accelerometry-based falls event detection. IEEE Transactions on Neural Systems and Rehabilitation Engineering 18(6), 619-627 (2010)

4. Bourke, A., O'Brien, J., Lyons, G.: Evaluation of a threshold-based triaxial accelerometer fall detection algorithm. Gait and Posture 26, 194-199 (2007)

5. Casilari, E., Santoyo-Ramn, J.A., Cano-Garca, J.M.: Umafall: A multisensor dataset for the research on automatic fall detection. Procedia Computer Science 110(Supplement C), 32 - 39 (2017), http://www.sciencedirect.com/science/ article/pii/S1877050917312899

6. Chawla, N.V., Bowyer, K.W., Hall, L.O., Kegelmeyer, W.P.: Smote: synthetic minority over-sampling technique. Journal of artificial intelligence research pp. 321357 (2002)

7. Daher, M., Diab, A., Najjar, M.E.B.E., Khalil, M.A., Charpillet, F.: Elder tracking and fall detection system using smart tiles. IEEE Sensors Journal 17(2), 469-479 (Jan 2017), http://ieeexplore.ieee.org/document/7733127/

8. Delahoz, Y.S., Labrador, M.A.: Survey on fall detection and fall prevention using wearable and external sensors. Sensors 14(10), 19806-19842 (2014), http://www . mdpi.com/1424-8220/14/10/19806/htm

9. Fang, Y.C., Dzeng, R.J.: A smartphone-based detection of fall portents for construction workers. Procedia Eng. 85, 147-156 (2014)

10. Fang, Y.C., Dzeng, R.J.: Accelerometer-based fall-portent detection algorithm for construction tiling operation. Autom. Constr. 84, 214-230 (2017)

11. González, S., Sedano, J., Villar, J.R., Corchado, E., Herrero, Á., Baruque, B.: Features and models for human activity recognition. Neurocomputing in press (2015)

12. Hakim, A., Huq, M.S., Shanta, S., Ibrahim, B.: Smartphone based data mining for fall detection: Analysis and design. Procedia Computer Science 105, 46-51 (2017), http://www.sciencedirect.com/science/article/pii/S1877050917302065

13. Huynh, Q.T., Nguyen, U.D., Irazabal, L.B., Ghassemian, N., Tran, B.Q.: Optimization of an accelerometer and gyroscope-based fall detection algorithm. Journal of Sensors 2015 (2015)

14. Igual, R., Medrano, C., Plaza, I.: Challenges, issues and trends in fall detection systems. BioMedical Engineering OnLine 12(66) (2013), http://www. biomedical-engineering-online.com/content/12/1/66 
15. Igual, R., Medrano, C., Plaza, I.: A comparison of public datasets for accelerationbased fall detection. Medical Engineering and Physics 37(9), 870-878 (2015), http: //www.sciencedirect.com/science/article/pii/S1350453315001575

16. Kangas, M., Konttila, A., Lindgren, P., Winblad, I., Jämsaä, T.: Comparison of low-complexity fall detection algorithms for body attached accelerometers. Gait and Posture 28, 285-291 (2008)

17. Khojasteh, S.B., Villar, J.R., de la Cal, E., González, V.M., Sedano, J., YAZG̈GN, H.R.: submitted to the 13th International Conference on Soft Computing Models in Industrial and Environmental Applications, chap. Evaluation of a Wrist-based Wearable Fall Detection Method (2018)

18. Kuhn, M.: The caret package. http://topepo.github.io/caret/index.html (2017), last checked 15-1-2018

19. Kumari, P., Mathew, L., Syal, P.: Increasing trend of wearables and multimodal interface for human activity monitoring: A review. Biosensors and Bioelectronics 90(15), 298-307 (Apr 2017)

20. Purch.com: Top ten reviews for fall detection of seniors. http://www . toptenreviews.com/health/senior-care/best-fall-detection-sensors/ (2018)

21. R Development Core Team: R: A Language and Environment for Statistical Computing. R Foundation for Statistical Computing, Vienna, Austria (2008), http://www.R-project.org, ISBN 3-900051-07-0

22. Sabatini, A.M., Ligorio, G., Mannini, A., Genovese, V., Pinna, L.: Prior-to- and post-impact fall detection using inertial and barometric altimeter measurements. IEEE Transactions on Neural Systems and Rehabilitation Engineering 24 (2016)

23. Sorvala, A., Alasaarela, E., Sorvoja, H., Myllyla, R.: A two-threshold fall detection algorithm for reducing false alarms. In: Proceedings of 2012 6th International Symposium on Medical Information and Communication Technology (ISMICT) (2012)

24. Vergara, P.M., de la Cal, E., Villar, J.R., González, V.M., Sedano, J.: An iot platform for epilepsy monitoring and supervising. Journal of Sensors 2017, 18 (2017)

25. Villar, J.R., González, S., Sedano, J., Chira, C., Trejo, J.M.: Human activity recognition and feature selection for stroke early diagnosis. In: Pan, J.S., Polycarpou, M.M., Wozniak, M., de Carvalho, A., Quintián, H., Corchado, E. (eds.) Hybrid Artificial Intelligent Systems, Lecture Notes in Computer Science, vol. 8073. Springer Berlin Heidelberg (2013)

26. Villar, J.R., González, S., Sedano, J., Chira, C., Trejo-Gabriel-Galán, J.M.: Improving human activity recognition and its application in early stroke diagnosis. International Journal of Neural Systems 25(4), 1450036-1450055 (2015)

27. Wu, F., Zhao, H., Zhao, Y., Zhong, H.: Development of a wearable-sensor-based fall detection system. International Journal of Telemedicine and Applications 2015, 11 (2015), https://www.hindawi.com/journals/ijta/2015/576364/

28. Zhang, S., Wei, Z., Nie, J., Huang, L., Wang, S., , Li, Z.: A review on human activity recognition using vision-based method. Journal of Healthcare Engineering 2017 (2017)

29. Zhang, T., Wang, J., Xu, L., Liu, P.: Fall detection by wearable sensor and one-class svm algorithm. In: Huang DS., Li K., I.G. (ed.) Intelligent Computing in Signal Processing and Pattern Recognition, Lecture Notes in Control and Information Systems, vol. 345, pp. 858-863. Springer Berlin Heidelberg (2006) 